\title{
Relatively Compact Sets in Variable Exponent Morrey Spaces on Metric Spaces
}

\author{
Rovshan A. Bandaliyev, Przemysław Górka, Vagif S. Guliyev and \\ Yoshihiro Sawano
}

\begin{abstract}
We study a characterization of the precompactness of sets in variable exponent Morrey spaces on bounded metric measure spaces. Totally bounded sets are characterized from several points of view for the case of variable exponent Morrey spaces over metric measure spaces. This characterization is new in the case of constant exponents.
\end{abstract}

Mathematics Subject Classification. Primary 46E30; Secondary 47A30, $26 \mathrm{D} 07$.

Keywords. Metric measure spaces, variable exponent Lebesgue spaces, Morrey spaces, compactness.

\section{Introduction}

In the present article, we investigate relatively compact sets in variable exponent Morrey spaces. In classical $L^{p}$-spaces, the celebrated Riesz-Kolmogorov theorem nicely characterizes the relatively compact sets $[22,30]$. The aim of the paper is to characterize precompact sets in variable exponent Morrey spaces on an arbitrary doubling metric measure space. Our characterization is new even in the constant exponent case. Let us mention some generalizations of the Riesz-Kolmogorov theorem. Rafeiro characterized precompact sets in variable exponent Lebesgue spaces on Euclidean spaces [29]. For example, we refer to $[3,10,11]$ for the characterization of precompact sets in variable exponent Lebesgue spaces on an arbitrary doubling metric measure space. Recently, the precompactness in quasi-Banach function spaces was studied in $[6,12,13]$ (see $[4,14]$ as well).

The study of variable exponent function spaces is now an extensively developed field due to the advent of two books $[7,8]$. Function spaces with variable exponents are a very active area of research and this theory finds many applications, e.g., in nonlinear elastic mechanics [36], electrorheological fluids [31], image restoration [24], and in differential equations with nonstandard growth. During the last decade, Lebesgue and Sobolev spaces with 
variable exponent have been intensively studied; see, for instance, the following survey papers $[9,32]$. On the other hand, there are also various advances in the study of variable exponent Morrey function spaces $[2,15,16,25]$. Those types of spaces have emerged very recently (we refer the reader to $[26,28]$ to see the classical setting). However, despite the significant progress in the theory of variable Lebesgue spaces, the study of variable exponent Morrey function spaces has a lot to be desired.

The present paper is structured as follows. In Sect. 2, we introduce the variable exponent Morrey norms and corresponding function spaces. We also recall standard results from the theory of variable exponent spaces including some facts on metric measure spaces. A characterization of relatively compact sets in variable exponent Morrey spaces over metric measure spaces is proved in Sect. 4. In the last section, we present some examples, remarks, and an open problem.

\section{Preliminaries}

\subsection{Variable Exponent Lebesgue and Morrey spaces}

We begin with the some definitions and some elementary facts. Let $(\Omega, \mu)$ be a $\sigma$-finite measure space. By a variable exponent, we mean a bounded $\mu$-measurable function $p(\cdot): \Omega \rightarrow(0, \infty)$. We denote the set of variable exponents on $\Omega$ by $\mathcal{P}(\Omega, \mu)$. For a measurable subset $U$ of $\Omega$, we put

$$
p_{+}(U)=\underset{x \in U}{\operatorname{ess} . \sup } p(x) ; \quad p_{-}(U)=\underset{x \in U}{\operatorname{ess} . i n f} p(x) .
$$

If $U=\Omega$, we will abbreviate $p_{ \pm}(U)$ to $p_{ \pm}$. In the present paper, we assume that the variable exponent function $p(\cdot)$ satisfies $0<p_{-} \leq p_{+}<\infty$.

The variable exponent Lebesgue space $L^{p(\cdot)}(\Omega)$ consists of all $\mu$-measurable functions $f: \Omega \rightarrow \mathbb{R}$ for which the modular

$$
\rho_{p(\cdot)}(f)=\int_{\Omega}|f(x)|^{p(x)} \mathrm{d} \mu(x)
$$

is finite. The space $L^{p(\cdot)}(\Omega)$ is a quasi-Banach space under the functional

$$
\|f\|_{L^{p(\cdot)}(\Omega)}=\inf \left\{\Lambda>0: \rho_{p(\cdot)}\left(\frac{f}{\Lambda}\right) \leq 1\right\}
$$

for $f \in L^{p(\cdot)}(\Omega)$. If the variable exponent $p(\cdot)$ is constant $p$, then $L^{p(\cdot)}(\Omega)$ is nothing but the classical Lebesgue space $L^{p}(\Omega)$. A fundamental observation made by Orlicz [27] is that the functional $\|\cdot\|_{L^{p(\cdot)}(\Omega)}$ given by (2.1) defines a norm in $L^{p(\cdot)}(\Omega)$ as long as $p_{-} \geq 1$. In particular, if $p_{-} \geq 1$, then $L^{p(\cdot)}(\Omega)$ is a Banach function spaces; see $[7,8,21]$. Variable Lebesgue space are realized as a special case of Musielak-Orlicz spaces.

In general, there are no functional relationships between the norm and the modular, but we have the following useful result: 
Lemma 2.1. $[7,8,21]$ Suppose that $p(\cdot) \in \mathcal{P}(\Omega, \mu)$ satisfies $0<p_{-} \leq p_{+}<\infty$, and let $f \in L^{p(\cdot)}(\Omega, \mu)$. Then

$$
\begin{aligned}
& \min \left\{\left(\rho_{p(\cdot)}(f)\right)^{\frac{1}{p_{-}}},\left(\rho_{p(\cdot)}(f)\right)^{\frac{1}{p_{+}}}\right\} \leq\|f\|_{L^{p(\cdot)}(\Omega)} \\
& \quad \leq \max \left\{\left(\rho_{p(\cdot)}(f)\right)^{\frac{1}{p_{-}}},\left(\rho_{p(\cdot)}(f)\right)^{\frac{1}{p_{+}}}\right\} .
\end{aligned}
$$

As the following lemma shows (see [3] for example), $L^{p(\cdot)}(\Omega)$ is a quasinormed space.

Lemma 2.2. Suppose that $p(\cdot) \in \mathcal{P}(\Omega, \mu)$ satisfies $0<p_{-} \leq p_{+}<\infty$. Then

$$
\|f+g\|_{L^{p(\cdot)}(\Omega)} \leq \max \left\{2^{\frac{1}{p_{-}}}, 2^{\frac{p_{+}}{p_{-}}}\right\}\left(\|f\|_{L^{p(\cdot)}(\Omega)}+\|g\|_{L^{p(\cdot)}(\Omega)}\right)
$$

for all $f, g \in L^{p(\cdot)}(\Omega)$. In particular

$$
\|f+g\|_{L^{p(\cdot)}(\Omega)} \leq 2^{\frac{1+p_{+}}{p_{-}}}\left(\|f\|_{L^{p(\cdot)}(\Omega)}+\|g\|_{L^{p(\cdot)}(\Omega)}\right) .
$$

We need the following lemma on the generalized Hölder inequality in variable exponent Lebesgue spaces.

Lemma 2.3 [19]. Let $\Omega$ be a measurable subset of $X$. Suppose that we have $p(\cdot), q(\cdot), s(\cdot) \in \mathcal{P}(\Omega, \mu)$ be such that

$$
\frac{1}{s(x)}=\frac{1}{p(x)}+\frac{1}{q(x)}
$$

for almost every $x \in \Omega$. Then

$$
\|f g\|_{L^{s(\cdot)}(\Omega)} \leq\left\{\left(\frac{s(\cdot)}{p(\cdot)}\right)_{+}+\left(\frac{s(\cdot)}{q(\cdot)}\right)_{+}\right\}\|f\|_{L^{p(\cdot)}(\Omega)}\|g\|_{L^{q(\cdot)}(\Omega)}
$$

holds for all $f \in L^{p(\cdot)}(\Omega)$ and $g \in L^{q(\cdot)}(\Omega)$.

Here and below, we work in a metric measure space $(X, \rho, \mu)$ equipped with a metric $\rho$ and the Borel regular measure $\mu$. Let $\lambda(\cdot): X \rightarrow[0,1]$ be a measurable function. The variable Morrey space $L^{p(\cdot), \lambda(\cdot)}(X)$ is introduced via the quasi-norm

$$
\|f\|_{L^{p(\cdot), \lambda(\cdot)(X)}}:=\sup _{\substack{x \in X \\ t>0}} \mu(B(x, t))^{-\frac{\lambda(x)}{p(x)}}\left\|f \chi_{B(x, t)}\right\|_{L^{p(\cdot)}(X)} .
$$

This is quasi-Banach spaces with respect to the quasi-norm $(2.2)$ (see $[2,17$, 18]).

\section{Remark 2.4 .}

1. The above definition naturally extends the classical one. Note that if $\lambda(\cdot):=0$, then $L^{p(\cdot), \lambda(\cdot)}(X)$ coincides with the variable Lebesgue space $L^{p(\cdot)}(X)$. Meanwhile, if $\lambda(\cdot)$ and $p(\cdot)$ are constant exponents $\lambda$ and $p$, respectively, then the variable Morrey space $L^{p(\cdot), \lambda(\cdot)}(X)$ coincides with the classical Morrey space $L^{p, \lambda}(X)[28]$. 
2. As is seen from the definition of $L^{p(\cdot), \lambda(\cdot)}(X)$, this function space depends on $p(\cdot)$ and $\lambda(\cdot) / p(\cdot)$. The function $p(\cdot)$ itself can be considered modulo the sets of $\mu$-measure zero. However, one can not do so for $\lambda(\cdot) / p(\cdot)$. A prominent example reflecting this fact is the following example: Let $(X, \rho, \mu)$ be a the unit ball in $\mathbb{R}^{d}$ with the Lebesgue measure, and let $p(\cdot):=2$ and $\lambda(\cdot)=\frac{1}{3}+\frac{1}{3} \chi_{X \cap \mathbb{Q}^{d}}$. Then, $L^{p(\cdot), \lambda(\cdot)}(X)$ coincides with $L^{2, \frac{1}{3}}(X) \cap L^{2, \frac{2}{3}}(X)$.

We carry over Lemma 2.1 to variable exponent Morrey spaces.

Lemma 2.5. Let $p(\cdot) \in \mathcal{P}(X, \mu)$ satisfy $0<p_{-} \leq p_{+}<\infty$, and let $\lambda(\cdot): X \rightarrow$ $[0,1]$ be an exponent. Then, for $f \in L^{p(\cdot), \lambda(\cdot)}(X)$

$$
\begin{aligned}
& \sup _{\substack{x \in X \\
t>0}} \mu(B(x, t))^{-\frac{\lambda(x)}{p(x)}} \min \left\{\left(\rho_{p(\cdot)}\left(f \chi_{B(x, t)}\right)\right)^{\frac{1}{p_{-}}},\left(\rho_{p(\cdot)}\left(f \chi_{B(x, t)}\right)\right)^{\frac{1}{p_{+}}}\right\} \\
& \quad \leq\|f\|_{L^{p(\cdot), \lambda(\cdot)}(X)} \\
& \quad \leq \sup _{\substack{x \in X \\
t>0}} \mu(B(x, t))^{-\frac{\lambda(x)}{p(x)}} \max \left\{\left(\rho_{p(\cdot)}\left(f \chi_{B(x, t)}\right)\right)^{\frac{1}{p_{-}}},\left(\rho_{p(\cdot)}\left(f \chi_{B(x, t)}\right)\right)^{\frac{1}{p_{+}}}\right\} .
\end{aligned}
$$

Proof. Let $\Omega=X$ in Lemma 2.1. By substituting $f \chi_{B(x, t)}$ into $f$, we have

$$
\begin{aligned}
& \min \left\{\left(\rho_{p(\cdot)}\left(f \chi_{B(x, t)}\right)\right)^{\frac{1}{p_{-}}},\left(\rho_{p(\cdot)}\left(f \chi_{B(x, t)}\right)\right)^{\frac{1}{p_{+}}}\right\} \leq\left\|f \chi_{B(x, t)}\right\|_{L^{p(\cdot)}(X)} \\
& \quad \leq \max \left\{\left(\rho_{p(\cdot)}\left(f \chi_{B(x, t)}\right)\right)^{\frac{1}{p_{-}}},\left(\rho_{p(\cdot)}\left(f \chi_{B(x, t)}\right)\right)^{\frac{1}{p_{+}}}\right\} .
\end{aligned}
$$

If we insert this estimate into the definition of $\|f\|_{L^{p(\cdot), \lambda(\cdot)(X)}}$, we obtain the desired result.

We transform Lemma 2.2 to the one in variable exponent Morrey spaces.

Lemma 2.6. Let $p(\cdot)$ and $\lambda(\cdot)$ be non-negative variable exponents, such that $0<p_{-} \leq p_{+}<\infty$ and that $\sup _{x \in X} \lambda(x) \leq 1$, and let $f, g \in L^{p(\cdot), \lambda(\cdot)}(\Omega)$. Then

$$
\|f+g\|_{L^{p(\cdot), \lambda(\cdot)}(\Omega)} \leq \max \left\{2^{\frac{1}{p_{-}}}, 2^{\frac{p_{+}}{p_{-}}}\right\}\left(\|f\|_{L^{p(\cdot), \lambda(\cdot)}(\Omega)}+\|g\|_{L^{p(\cdot), \lambda(\cdot)}(\Omega)}\right) .
$$

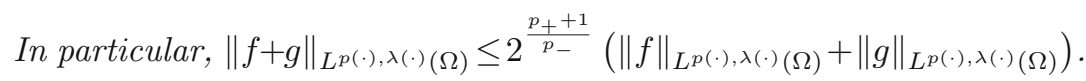

The following two lemmata will be important in variable exponent Morrey spaces.

Lemma 2.7. Let $(X, \rho, \mu)$ be a metric measure space with finite measure, and let $p(\cdot)$ and $\lambda(\cdot)$ be non-negative variable exponents. Then

$$
L^{p(\cdot), \lambda(\cdot)}(X) \hookrightarrow L^{p(\cdot)}(X) .
$$

More precisely, for all $f \in L^{p(\cdot)}(X)$

$$
\|f\|_{L^{p(\cdot)}(X)} \leq \max \left\{\mu(X)^{a}, \mu(X)^{b}\right\}\|f\|_{L^{p(\cdot), \lambda(\cdot)(X)}},
$$


where $a:=\sup _{x \in X} \frac{\lambda(x)}{p(x)}$ and $b:=\inf _{x \in X} \frac{\lambda(x)}{p(x)}$.

Proof. We have only to prove (2.3); the embedding is its direct consequence. We calculate

$$
\begin{aligned}
\|f\|_{L^{p(\cdot)}(X)} & =\sup _{\substack{x \in X \\
t>0}} \frac{\mu(B(x, t))^{\frac{\lambda(x)}{p(x)}}}{\mu(B(x, t))^{\frac{\lambda(x)}{p(x)}}}\|f\|_{L^{p(\cdot)}(B(x, t))} \\
& \leq \sup _{\substack{x \in X \\
t>0}} \mu(B(x, t))^{\frac{\lambda(x)}{p(x)}} \sup _{\substack{x \in X \\
t>0}} \mu(B(x, t))^{-\frac{\lambda(x)}{p(x)}}\left\|f \chi_{B(x, t)}\right\|_{L^{p(\cdot)}(X)} \\
& \leq \sup _{x \in X} \mu(X)^{\frac{\lambda(x)}{p(x)}}\|f\|_{L^{p(\cdot), \lambda(\cdot)}(X)} \\
& =\max _{x}\left\{\mu(X)^{a}, \mu(X)^{b}\right\}\|f\|_{L^{p(\cdot), \lambda(\cdot)(X)},}
\end{aligned}
$$

as required.

Under an additional conditions $\sup _{x \in X} \frac{\lambda(x)}{p(x)} \leq \frac{1}{p_{+}}$and $\mu(X)<\infty$, we

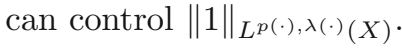

Lemma 2.8. Let $(X, \rho, \mu)$ be a metric measure space with finite measure, and let $p(\cdot)$ and $\lambda(\cdot)$ be non-negative variable exponents. Assume $0<p_{-} \leq p_{+}<$ $\infty$ and $\sup _{x \in X} \frac{\lambda(x)}{p(x)} \leq \frac{1}{p_{+}}$. Then

$$
\|1\|_{L^{p(\cdot), \lambda(\cdot)}(X)} \leq \max \left\{\mu(X)^{\frac{1}{p_{+}}-\sup _{x \in X} \frac{\lambda(x)}{p(x)}}, \mu(X)^{\frac{1}{p_{-}}-\inf _{x \in X} \frac{\lambda(x)}{p(x)}}\right\} .
$$

More generally, for any measurable set $F$

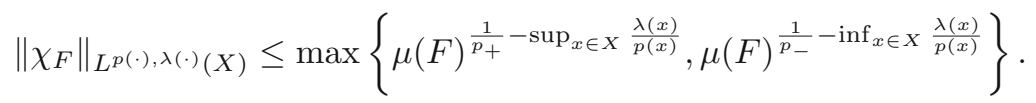

Proof. Thanks to Lemma 2.1, we obtain

$$
\begin{aligned}
\| \chi_{F} & \|_{L^{p(\cdot), \lambda(\cdot)}(X)} \\
= & \sup _{\substack{x \in X \\
t>0}} \mu(B(x, t))^{-\frac{\lambda(x)}{p(x)}}\left\|\chi_{B(x, t) \cap F}\right\|_{L^{p(\cdot)}(X)} \\
\leq & \sup _{\substack{x \in X \\
t>0}} \mu(B(x, t))^{-\frac{\lambda(x)}{p(x)}} \max \left\{\mu(B(x, t) \cap F)^{\frac{1}{p_{-}}}, \mu(B(x, t) \cap F)^{\frac{1}{p_{+}}}\right\} \\
\leq & \sup _{\substack{x \in X \\
t>0}} \max \left\{\mu(B(x, t) \cap F)^{\frac{1}{p_{-}}-\frac{\lambda(x)}{p(x)}}, \mu(B(x, t) \cap F)^{\frac{1}{p_{+}}-\frac{\lambda(x)}{p(x)}}\right\} .
\end{aligned}
$$

Recall that $\sup _{x \in X} \frac{\lambda(x)}{p(x)} \leq \frac{1}{p_{+}}$by assumption and hence $\frac{1}{p_{ \pm}}-\frac{\lambda(x)}{p(x)} \geq 0$ for all $x \in X$. Thus

$$
\left\|\chi_{F}\right\|_{L^{p(\cdot), \lambda(\cdot)}(X)} \leq \sup _{x \in X} \max \left\{\mu(F)^{\frac{1}{p_{-}}-\frac{\lambda(x)}{p(x)}}, \mu(F)^{\frac{1}{p_{+}}-\frac{\lambda(x)}{p(x)}}\right\} .
$$


It remains to use $\sup _{x \in X} \mu(F)^{-\frac{\lambda(x)}{p(x)}}=\max \left\{\mu(F)^{-\sup _{x \in X} \frac{\lambda(x)}{p(x)}}, \mu(F)^{-\inf _{x \in X} \frac{\lambda(x)}{p(x)}}\right\}$.

Remark 2.9. Let $(X, \rho, \mu)$ be a metric measure space with finite measure, and let $p(\cdot)$ and $\lambda(\cdot)$ be non-negative variable exponents. Assume $0<p_{-} \leq$ $p_{+}<\infty$ and $\sup _{x \in X} \frac{\lambda(x)}{p(x)}<\frac{1}{p_{+}}$. Then, the constant function 1 belongs to $L^{p(\cdot), \lambda(\cdot)}(X)$. Moreover, for any $\varepsilon$ there exists $\delta>0$, such that $\left\|\chi_{E}\right\|_{L^{p(\cdot), \lambda(\cdot)}(X)}$ $<\varepsilon$ for any measurable set $E$ satisfying $\mu(E)<\delta$.

\subsection{Log-Hölder Continuity of Functions}

Let $(X, \rho)$ be a metric space, and let $\Omega$ be a measurable subset of $X$. We say that a function $p(\cdot): \Omega \rightarrow \mathbb{R}$ is locally log-Hölder-continuous on $\Omega$, if there exists $C_{1}>0$, such that for all $x, y \in \Omega$

$$
|p(x)-p(y)| \leq \frac{C_{1}}{\log \left(e+\rho(x, y)^{-1}\right)} .
$$

In addition, we say that the exponent $p(\cdot)$ satisfies the log-Hölder decay condition at infinity with a fixed point $x_{0} \in X$, if there exist $p_{\infty}, C_{2}>0$, such that for all $x \in \Omega$

$$
\left|p(x)-p_{\infty}\right| \leq \frac{C_{2}}{\log \left(e+\rho\left(x, x_{0}\right)\right)} .
$$

We also say that $p(\cdot)$ is globally log-Hölder-continuous on $\Omega$ if it is locally log-Hölder-continuous on and satisfies the log-Hölder decay condition at infinity. Then, the constant

$$
C_{\log }(p(\cdot)):=\max \left\{C_{1} ; C_{2}\right\}
$$

is called the log-Hölder constant for the exponent $p(\cdot)$. We also define the set of log-Hölder-continuous exponents by

$$
\mathcal{P}_{\log }(\Omega, \mu):=\left\{p(\cdot) \in \mathcal{P}(\Omega, \mu): \frac{1}{p(\cdot)} \text { is globally log-Hölder-continuous }\right\} .
$$

\subsection{Metric Measure Spaces}

Let $(X, \rho, \mu)$ be a metric measure space equipped with a metric $\rho$ and the Borel regular measure $\mu$. We assume throughout the paper that the measure of every open nonempty set is positive and that the measure of every bounded set is finite. Additionally, we assume that the measure $\mu$ satisfies a doubling condition. It means that there exists a constant $C_{\mu}>0$, such that for every ball $B(x, r)$

$$
\mu(B(x, 2 r)) \leq C_{\mu} \mu(B(x, r)) .
$$

It is well known (see [20] for example) that the doubling condition implies that there exists a positive constant $D$ satisfying

$$
\frac{\mu\left(B\left(x_{2}, r_{2}\right)\right)}{\mu\left(B\left(x_{1}, r_{1}\right)\right)} \leq D\left(\frac{r_{2}}{r_{1}}\right)^{s}
$$

where $s:=\log _{2} C_{\mu}$ for all balls $B\left(x_{2}, r_{2}\right)$ and $B\left(x_{1}, r_{1}\right)$, with $r_{2} \geq r_{1}>0$ and $x_{1} \in B\left(x_{2}, r_{2}\right)$. It follows from the above inequality that if we fix a 
ball $B\left(x_{0}, R\right)$, then there exists $b>0$, such that the following lower Ahlfors condition holds for $x \in B\left(x_{0}, R\right)$ and for $r \leq R$ :

$$
\mu(B(x, r)) \geq b r^{s} .
$$

In particular, if $X$ is bounded, then there exists $b>0$, such that the following inequality holds for $r<\operatorname{diam}(X)$ and $x \in X$

$$
\mu(B(x, r)) \geq b r^{s} .
$$

Furthermore, we define $(f)_{A}$ the integral average of the $\mu$-measurable function $f$ over the measurable set $A$; that is

$$
(f)_{A}:=f_{A} f(x) \mathrm{d} \mu(x)=\frac{1}{\mu(A)} \int_{A} f(x) \mathrm{d} \mu(x) .
$$

\subsection{Space of Measurable Functions $L^{0}(X)$}

Let $(X, \mu)$ be a finite measure space. ${ }^{1}$ Then, by $L^{0}(X)$, we denote the space of measurable functions on $X$. This space is a complete metric space with respect to the metric

$$
d_{0}(f ; g):=\int_{X} \phi(f(x)-g(x)) \mathrm{d} \mu(x),
$$

where

$$
\phi(t):=\frac{|t|}{1+|t|} \quad(t \in \mathbb{C}) .
$$

It is well known that the convergence in this metric is equivalent to the convergence in measure. Using the idea employed in [23], one can show the following theorem.

Theorem 2.10. Let $(X, \rho, \mu)$ be a totally bounded metric measure space. Any almost uniformly bounded and almost equicontinuous subset of $L^{0}(X, \mu)$ is totally bounded. That is, a subset $\mathcal{F}$ of $L^{0}(X, \mu)$ is totally bounded if for any $\varepsilon>0$ and any function $f \in \mathcal{F}$, there exists measurable subset $E(f) \subset X$ and constants $\delta>0$ and $\Lambda>0$ independent of $f$ with the following properties:

(1) $\mu(E(f))<\varepsilon$;

(2) $|f(x)-f(y)|<\varepsilon$ for any $x, y \in X \backslash E(f)$ satisfying $\rho(x, y)<\delta$;

(3) $|f(x)| \leq \Lambda$ for $x \in X \backslash E(f)$.

\section{Totally Bounded Sets in $L^{p(\cdot), \lambda(\cdot)}(X)$}

We go back to a measure space $(X, \rho, \mu)$. In this section, we study totally bounded sets in $L^{p(\cdot), \lambda(\cdot)}(X)$, where $(X, \rho, \mu)$ is a doubling metric space. We start with the following version of the Lebesgue-Vitali compactness-type theorem in $L^{p(\cdot), \lambda(\cdot)}(X)$ over finite metric measure spaces.

Theorem 3.1. Let $(X, \rho, \mu)$ be a metric measure space with finite measure. Let $p(\cdot)$ and $\lambda(\cdot)$ be non-negative variable exponents, such that $0<p_{-} \leq p_{+}<\infty$ and that $\sup _{x \in X} \frac{\lambda(x)}{p(x)} \leq \frac{1}{p_{+}}$. Assume that a subset $\mathcal{F}$ of $L^{p(\cdot), \lambda(\cdot)}(X)$ satisfies the following conditions:

\footnotetext{
${ }^{1}$ Finite measure space means that the measure of the space is finite.
} 
(i) $\mathcal{F}$ is totally bounded in $L^{0}(X)$;

(ii) the family $\mathcal{F}$ is $L^{p(\cdot), \lambda(\cdot)}(X)$-equi-integrable, that is, for all $\varepsilon>0$, there exists $\delta>0$, such that $\sup _{f \in \mathcal{F}}\left\|f \chi_{A}\right\|_{L^{p(\cdot), \lambda(\cdot)(X)}}<\varepsilon$ for all $A \subset X$ satisfying $\mu(A)<\delta$.

Then, $\mathcal{F}$ is totally bounded in $L^{p(\cdot), \lambda(\cdot)}(X)$.

Proof. We fix $\varepsilon>0$. We choose $\alpha \in(0,1)$, such that $\alpha\|1\|_{L^{p(\cdot), \lambda(\cdot)}(X)}<$ $2^{\frac{p_{+}+1}{p_{-}}} \varepsilon$. Then, thanks to $(i i)$, there exists $\delta>0$, such that $\sup _{f \in \mathcal{F}}\left\|f \chi_{A}\right\|_{L^{p(\cdot), \lambda(\cdot)(X)}}<\varepsilon$, whenever $A$ is a measurable set satisfying $\mu(A)<\delta$. Thanks to $(i)$, there exists a $\delta \phi(\alpha)$-net $\left\{f_{k}\right\}_{k \in K} \subset \mathcal{F}$ in $L^{0}(X)$, where $\phi$ is given by (2.6) and $K$ is a finite set.

By the definition of $\delta \phi(\alpha)$-nets, for any $f \in \mathcal{F}$, there exists $k \in K$, such that $d_{0}\left(f, f_{k}\right)<\delta \phi(\alpha)$. Let

$$
X_{\alpha}:=\left\{x \in X:\left|f-f_{k}\right| \leq \alpha\right\},
$$

and let

$$
X_{\alpha}^{\star}:=X \backslash X_{\alpha}
$$

Therefore, thanks to Lemma 2.6, we obtain

$$
\begin{aligned}
\| f & -f_{k} \|_{L^{p(\cdot), \lambda(\cdot)}(X)} \\
& =\left\|\left(f-f_{k}\right)\left(\chi_{X_{\alpha}}+\chi_{X_{\alpha}^{\star}}\right)\right\|_{L^{p(\cdot), \lambda(\cdot)}(X)} \\
& \leq 2^{\frac{p_{+}+1}{p_{-}}}\left(\left\|\left(f-f_{k}\right) \chi_{X_{\alpha}}\right\|_{L^{p(\cdot), \lambda(\cdot)}(X)}+\left\|\left(f-f_{k}\right) \chi_{X_{\alpha}^{\star}}\right\|_{L^{p(\cdot), \lambda(\cdot)}(X)}\right) .
\end{aligned}
$$

Since $f, f_{k} \in \mathcal{F}$

$$
\begin{aligned}
\| f & -f_{k} \|_{L^{p(\cdot), \lambda(\cdot)}(X)} \\
& \leq 2^{\frac{p_{+}+1}{p_{-}}}\left(\alpha\left\|\chi_{X_{\alpha}}\right\|_{L^{p(\cdot), \lambda(\cdot)}(X)}+2^{\frac{p_{+}+1}{p_{-}}+1} \sup _{g \in \mathcal{F}}\left\|g \chi_{X_{\alpha}^{\star}}\right\|_{L^{p(\cdot), \lambda(\cdot)}(X)}\right) \\
& \leq 2^{\frac{p_{+}+1}{p_{-}}}\left(\alpha\|1\|_{L^{p(\cdot), \lambda(\cdot)(X)}}+2^{\frac{p_{+}+1}{p_{-}}+1} \sup _{g \in \mathcal{F}}\left\|g \chi_{X_{\alpha}^{\star}}\right\|_{L^{p(\cdot), \lambda(\cdot)}(X)}\right)
\end{aligned}
$$

thanks to Lemma 2.8 once again. Moreover, in view of the monotonicity of $\phi$ and the Markov inequality, ${ }^{2}$ we deduce

$$
\begin{aligned}
\mu\left(X_{\alpha}^{\star}\right) & =\mu\left(\left\{x \in X: \phi\left(\left|f(x)-f_{k}(x)\right|\right)>\phi(\alpha)\right\}\right) \\
& \leq \frac{1}{\phi(\alpha)} \int_{X} \phi\left(\left|f(x)-f_{k}(x)\right|\right) \mathrm{d} \mu(x) \\
& =\frac{d_{0}\left(f, f_{k}\right)}{\phi(\alpha)}<\delta .
\end{aligned}
$$

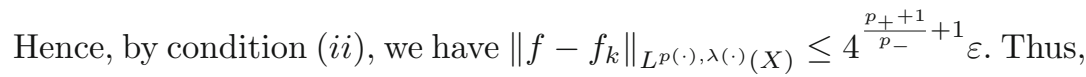
$\mathcal{F}$ is totally bounded in $L^{p(\cdot), \lambda(\cdot)}(X)$.

\footnotetext{
${ }^{2}$ For $\epsilon>0$ and a measurable function $g$, the following inequality $\mu(\{x \in X:|g(x)|>\epsilon\}) \leq$ $\frac{1}{\epsilon} \int_{X}|g(x)| \mathrm{d} \mu(x)$ holds.
} 
Now, we prove the converse. We aim to find additional conditions on variable Morrey spaces that guarantee the validity of $(i)$ and $(i i)$.

Now, we recall the definition of the absolute continuity.

Definition 3.2. We say that an element $f$ in a quasi-Banach space $B \subset L^{0}(X)$ has absolutely continuous quasi-norm if $\lim _{j \rightarrow \infty}\left\|f \chi_{E_{j}}\right\|_{B}=0$ for every sequence $\left\{E_{j}\right\}_{j=1}^{\infty}$ of measurable sets satisfying $\lim _{j \rightarrow \infty} \chi_{E_{j}}=0 \mu$-almost everywhere.

We use the condition of the absolute continuity as follows:

Lemma 3.3. Let $(X, \rho, \mu)$ be a metric measure space and $x_{0} \in X$. If an element $f$ in a quasi-Banach space $B \subset L^{0}(X)$ has absolutely continuous quasinorm, then

$$
\lim _{R \rightarrow \infty}\left\|f-\chi_{B\left(x_{0}, R\right)} \chi_{(0, R)}(|f|) f\right\|_{B}=0 .
$$

Proof. Simply observe that $\chi_{X \backslash B\left(x_{0}, R\right) \cap\{|f| \leq R\}} \rightarrow 0$ as $R \rightarrow \infty$.

Motivated by [34], the set of all functions in $L^{p(\cdot), \lambda(\cdot)}(X)$ having absolutely continuous quasi-norm is denoted by $\widetilde{L}^{p(\cdot), \lambda(\cdot)}(X)$. We equip $L^{p(\cdot), \lambda(\cdot)}(X)$ with the norm $\|f\|_{\widetilde{L}^{p(\cdot), \lambda(\cdot)}(X)}=\|f\|_{L^{p(\cdot), \lambda(\cdot)(X)}}$ for $f \in L^{p(\cdot), \lambda(\cdot)}(X)$.

Theorem 3.4. Let $(X, \rho, \mu)$ be a metric measure space with finite measure and let $p(\cdot)$ and let $\lambda(\cdot)$ be non-negative variable exponents, such that $0<p_{-} \leq$ $p_{+}<\infty$. Then, conditions $(i)$ and $($ ii $)$ of Theorem 3.1 hold for any subset $\overline{\mathcal{F}}$ of $\widetilde{L}^{p(\cdot), \lambda(\cdot)}(X)$ which is totally bounded in $\widetilde{L}^{p(\cdot), \lambda(\cdot)}(X)$.

Proof. We will verify $(i)$. Write $A:=\max \left\{\mu(X)^{a}, \mu(X)^{b}\right\}$. Let us fix $\varepsilon>0$ and $\alpha \in(0,1)$ such that $\phi(\alpha) \mu(X) \leq \varepsilon$, where $\phi$ is as in (2.6). Additionally, choose an $\varepsilon \alpha$-net $\left\{f_{k}\right\}_{k=1}^{n}$ in $\widetilde{L}^{p(\cdot), \lambda(\cdot)}(X)$. Then, by the definition of $\varepsilon \alpha$-nets, for any $f \in \mathcal{F}$, there exists $k \in\{1, \ldots, n\}$, such that $\left\|f-f_{k}\right\|_{\widetilde{L}^{p(\cdot), \lambda(\cdot)}(X)} \leq$ $\varepsilon \alpha$. Hence, due to Lemma 2.7, we have

$$
\left\|f-f_{k}\right\|_{L^{p(\cdot)}(X)} \leq A\left\|f-f_{k}\right\|_{L^{p(\cdot), \lambda(\cdot)}(X)} \leq A \varepsilon \alpha .
$$

Thus

$$
\left\|\frac{f-f_{k}}{\alpha}\right\|_{L^{p(\cdot)}(X)} \leq A \varepsilon .
$$

Let $X_{\alpha}$ and $X_{\alpha}^{\star}$ be the sets defined by (3.1) and (3.2), respectively. Then, from the property of $\alpha$, we obtain

$$
\begin{aligned}
d_{0}\left(f, f_{k}\right) & =\int_{X_{\alpha}} \phi\left(f(x)-f_{k}(x)\right) \mathrm{d} \mu(x)+\int_{X_{\alpha}^{*}} \phi\left(f(x)-f_{k}(x)\right) \mathrm{d} \mu(x) \\
& \leq \phi(\alpha) \mu(X)+\int_{X_{\alpha}^{*}} \mathrm{~d} \mu(x) \\
& \leq \varepsilon+\int_{X_{\alpha}^{*}} \mathrm{~d} \mu(x) .
\end{aligned}
$$


By Lemma 2.1, we have

$$
\begin{aligned}
\int_{X_{\alpha}^{*}} \mathrm{~d} \mu(x) & \leq \int_{X_{\alpha}^{\star}} \frac{\left|f(x)-f_{k}(x)\right|^{p(x)}}{\alpha^{p(x)}} \mathrm{d} \mu(x) \\
& \leq \int_{X} \frac{\left|f(x)-f_{k}(x)\right|^{p(x)}}{\alpha^{p(x)}} \mathrm{d} \mu(x) \\
& \leq \max \left\{\left\|\frac{f-f_{k}}{\alpha}\right\|_{L^{p(\cdot)}(X)}^{p_{-}},\left\|\frac{f-f_{k}}{\alpha}\right\|_{L^{p(\cdot)}(X)}^{p_{+}}\right\} \\
& \leq \max \left\{(A \varepsilon)^{p_{-}},(A \varepsilon)^{p_{+}}\right\} .
\end{aligned}
$$

We write $\varepsilon_{0}=\varepsilon_{0}(\varepsilon):=\varepsilon+\max \left\{(A \varepsilon)^{p_{-}},(A \varepsilon)^{p_{+}}\right\}$. Then, the above inequality reads as $d_{0}\left(f, f_{k}\right) \leq \varepsilon_{0}$. This implies that $\left\{f_{k}\right\}_{k=1}^{n}$ is an $\varepsilon_{0}$-net. Since $\lim _{\varepsilon \rightarrow 0} \varepsilon_{0}=\lim _{\varepsilon \rightarrow 0} \varepsilon_{0}(\varepsilon)=0$, we see that $(i)$ is satisfied.

We will verify $(i i)$. We fix $\varepsilon>0$ and choose an $\varepsilon$-net $\left\{f_{k}\right\}_{k=1}^{n} \subset \mathcal{F}$ in $L^{p(\cdot), \lambda(\cdot)}(X)$. Fix $k=0,1, \ldots, n$. Since $f_{k} \in \widetilde{L}^{p(\cdot), \lambda(\cdot)}(X)$, there exists $\delta_{k}$, such that $\left\|f_{k} \chi_{A}\right\|_{\widetilde{L}^{p(\cdot), \lambda(\cdot)}(X)}<\varepsilon$ as long as a measurable subset $A$ satisfies $\mu(A)<\delta_{k}$. We now move $k$. For $A \subset X$ satisfying $\mu(A)<\delta:=$ $\min \left\{\delta_{k}: k=0,1, \ldots, n\right\}$, we have

$$
\left\|f_{k} \chi_{A}\right\|_{\widetilde{L}^{p(\cdot), \lambda(\cdot)(X)}}<\varepsilon .
$$

For any $f \in \mathcal{F}$, there exists $k$, such that $\left\|f-f_{k}\right\|_{L^{p(\cdot), \lambda(\cdot)}(X)} \leq \varepsilon$, since $\left\{f_{k}\right\}_{k=1}^{n}$ is an $\varepsilon$-net in $L^{p(\cdot), \lambda(\cdot)}(X)$. Therefore, we obtain

$$
\begin{aligned}
\left\|f \chi_{A}\right\|_{L^{p(\cdot), \lambda(\cdot)(X)}} & \leq 2^{\frac{p_{+}+1}{p_{-}}}\left(\left\|\left(f-f_{k}\right) \chi_{A}\right\|_{\widetilde{L}^{p(\cdot), \lambda(\cdot)(X)}}+\left\|f_{k} \chi_{A}\right\|_{L^{p(\cdot), \lambda(\cdot)}(X)}\right) \\
& \leq 2^{\frac{p_{+}+1}{p_{-}}+1} \varepsilon .
\end{aligned}
$$

Consequently, $\mathcal{F}$ satisfies $($ ii $)$.

From Theorems 3.1 and 3.4, we have the following corollaries.

Corollary 3.5. Let $(X, \rho, \mu)$ be a metric measure space with finite measure and let $p(\cdot) \in \mathcal{P}(X, \mu)$ satisfy $0<p_{-} \leq p_{+}<\infty$. Let also $\lambda(\cdot): X \rightarrow[0,1]$ be a non-negative exponent, such that $\sup _{x \in X} \frac{\lambda(x)}{p(x)} \leq \frac{1}{p_{+}}$. Then, a subset $\mathcal{F}$ of $\widetilde{L}^{p(\cdot), \lambda(\cdot)}(X)$ is totally bounded in $\widetilde{L}^{p(\cdot), \lambda(\cdot)}(X)$ if and only if the following conditions are satisfied:

(i) $\mathcal{F}$ is totally bounded in $L^{0}(X)$;

(ii) the family $\mathcal{F}$ is $\widetilde{L}^{p(\cdot), \lambda(\cdot)}(X)$-equi-integrable, that is, for all $\varepsilon>0$, there exists $\delta>0$, such that $\sup _{f \in \mathcal{F}}\left\|f \chi_{A}\right\|_{L^{p(\cdot), \lambda(\cdot)}(X)}<\varepsilon$ for any measurable set $A$ of $X$ with $\mu(A)<\delta$.

Let $\lambda(\cdot):=0$. In this case, Theorem 3.1 boils down to the LebesgueVitali compactness criteria in variable Lebesgue spaces, since $\widetilde{L}^{p(\cdot), \lambda(\cdot)}(X)=$ $L^{p(\cdot)}(X)$. We say that the family $\mathcal{F}$ is $p(\cdot)$-equi-integrable if for all $\varepsilon>0$, there exists $\delta>0$, such that $\sup _{f \in \mathcal{F}}\left\|f \chi_{A}\right\|_{L^{p(\cdot)}(X)}<\varepsilon$ for any measurable set $A$ of $X$ with $\mu(A)<\delta$. 
Corollary 3.6. [3] Let $(X, \mu)$ be a finite measure space, and let $p(\cdot) \in \mathcal{P}(X, \mu)$ satisfy $0<p_{-} \leq p_{+}<\infty$. Then, a subset $\mathcal{F}$ of $L^{p(\cdot)}(X)$ is totally bounded in $L^{p(\cdot)}(X)$ if and only if $\mathcal{F}$ is totally bounded in $L^{0}(X)$ and the family $\mathcal{F}$ is $p(\cdot)$-equi-integrable.

Lemma 3.7. Let $(X, \rho, \mu)$ be a doubling metric measure space with finite measure, and let $q \in\left(0, p_{-}\right)$. Assume that $\sup _{x \in X} \frac{\lambda(x)}{p(x)}<\frac{1}{p_{+}}$. Then, for $f \in L^{\infty}(X)$, we have

$$
\lim _{r \rightarrow+0}\left\|f_{B(\cdot, r)}|f(\cdot)-f(y)|^{q} d \mu(y)\right\|_{L^{\frac{p(\cdot)}{q}, \lambda(\cdot)}(X)}=0 .
$$

Proof. Let $r_{n}$ be a sequence such that $r_{n} \rightarrow 0$. We fix $\varepsilon>0$. Then thanks to Lemma 2.8, there exists $\delta>0$, such that $\left\|\chi_{E}\right\|_{L^{p(\cdot), \lambda(\cdot)}(X)}<\varepsilon$ for any measurable set $E$ satisfying $\mu(E)<\delta$.

Since $\mu$ is doubling, we have

$$
\lim _{r \rightarrow+0} f_{B(x, r)}|f(x)-f(y)|^{q} \mathrm{~d} \mu(y)=0
$$

for $\mu$-almost all $x \in X$. By the Egoroff Theorem, there exist $N_{0} \in N$ and a measurable set $F$, such that

$$
\sup _{x \in X \backslash F} f_{B\left(x, r_{n}\right)}|f(x)-f(y)|^{q} \mathrm{~d} \mu(y)<\varepsilon, \quad \mu(F)<\delta
$$

as long as $n \geq N_{0}$. For this $F$, we have

$$
f_{B\left(x, r_{n}\right)}|f(x)-f(y)|^{q} \mathrm{~d} \mu(y) \leq \varepsilon \chi_{X \backslash F}(x)+\left(2\|f\|_{L^{\infty}(X)}\right)^{q} \chi_{F}(x) .
$$

If we take the $L^{p(\cdot), \lambda(\cdot)}(X)$-norm, then we obtain

$$
\begin{aligned}
& \left\|f_{B\left(\cdot, r_{n}\right)}|f(\cdot)-f(y)|^{q} \mathrm{~d} \mu(y)\right\|_{L^{\frac{p(\cdot)}{q}, \lambda(\cdot)}(X)} \\
& \quad \leq \varepsilon\left\|\chi_{X \backslash F}\right\|_{L^{\frac{p(\cdot)}{q}, \lambda(\cdot)}(X)}+\left(2\|f\|_{L^{\infty}(X)}\right)^{q}\left\|\chi_{F}\right\|_{L^{\frac{p(\cdot)}{q}, \lambda(\cdot)}(X)} \\
& \quad \leq \varepsilon\|1\|_{L^{\frac{p(\cdot)}{q}, \lambda(\cdot)(X)}}+\left(2\|f\|_{L^{\infty}(X)}\right)^{q} \varepsilon^{q}
\end{aligned}
$$

as long as $n \geq N_{0}$. Since $\varepsilon>0$ is arbitrary, we obtain the desired result.

Let $q>0$ and $f \in L^{0}(X)$. The Hardy-Littlewood maximal operator $\mathcal{M}$ is defined by

$$
\mathcal{M}(f)(x):=\sup _{t>0} \frac{1}{\mu(B(x, t))} \int_{B(x, t)}|f(y)| \mathrm{d} \mu(y) .
$$

and the powered Hardy-Littlewood maximal operator is defined by

$$
\begin{aligned}
& \mathcal{M}_{q}(f)(x):=\left[\mathcal{M}\left(|f(\cdot)|^{q}\right)(x)\right]^{\frac{1}{q}} \\
& =\sup _{t>0}\left(\frac{1}{\mu(B(x, t))} \int_{B(x, t)}|f(y)|^{q} \mathrm{~d} \mu(y)\right)^{\frac{1}{q}} \quad(x \in X) .
\end{aligned}
$$


Theorem 3.8. Let $(X, \rho, \mu)$ be a doubling metric measure space with finite measure. Let $q \in\left(0, p_{-}\right)$and $\sup _{x \in X} \frac{\lambda(x)}{p(x)}<\frac{1}{p_{+}}$. Assume that the powered maximal operator $\mathcal{M}_{q}: L^{p(\cdot), \lambda(\cdot)}(X) \rightarrow L^{p(\cdot), \lambda(\cdot)}(X)$ is bounded. Then, we have the following properties for any subset $\mathcal{F}$ of $\widetilde{L}^{p(\cdot), \lambda(\cdot)}(X)$ which is totally bounded in $\widetilde{L}^{p(\cdot), \lambda(\cdot)}(X)$ :

(a) $\mathcal{F}$ is bounded in $L^{p(\cdot), \lambda(\cdot)}(X)$;

(b) $\lim _{r \rightarrow+0} \sup _{f \in \mathcal{F}}\left\|f_{B(\cdot, r)}|f(\cdot)-f(y)|^{q} d \mu(y)\right\|_{L^{\frac{p(\cdot)}{q}, \lambda(\cdot)}(X)}=0$;

(c) for some $x_{0} \in X, \lim _{R \rightarrow \infty} \sup _{f \in \mathcal{F}}\left\|f \chi_{X \backslash B\left(x_{0}, R\right)}\right\|_{L^{p(\cdot), \lambda(\cdot)}(X)}=0$.

Proof. Before the proof, we need a setup. Denote by $C$ the operator norm of $\mathcal{M}_{q}$ acting on $L^{p(\cdot), \lambda(\cdot)}(X)$. Assume that $\mathcal{F}$ is totally bounded in $\widetilde{L}^{p(\cdot), \lambda(\cdot)}(X)$. We fix $\varepsilon>0$ and let $\left\{f_{k}\right\}$ is an $\varepsilon$-net in $\mathcal{F}$. For each $f \in \mathcal{F}$, we choose $k \in\{1, \ldots, N\}$ satisfying

$$
\mathrm{I}_{1}:=\left\|f_{k}-f\right\|_{\widetilde{L}^{p(\cdot), \lambda(\cdot)(X)}}<\varepsilon .
$$

Put $M_{1}:=\sup _{k=1, \ldots, N}\left\|f_{k}\right\|_{\widetilde{L}^{p(\cdot), \lambda(\cdot)(X)}}$. We write

$$
\begin{aligned}
& \mathrm{I}_{2}:=\left\|f_{B(\cdot, r)}\left|f_{k}(y)-f_{k}(\cdot)\right|^{q} \mathrm{~d} \mu(y)\right\|_{\widetilde{L}^{\frac{p(\cdot)}{q}, \lambda(\cdot)}(X)}^{\frac{1}{q}}, \\
& \mathrm{I}_{3}:=\left\|f_{B(\cdot, r)}\left|f_{k}(y)-f(y)\right|^{q} \mathrm{~d} \mu(y)\right\|_{\widetilde{L}^{\frac{p(\cdot)}{q}, \lambda(\cdot)}(X)}^{\frac{1}{q}} .
\end{aligned}
$$

With this in mind, we prove $(a)-(c)$.

(a) Let $f \in \mathcal{F}$. Then, from Lemma 2.6, we deduce

$$
\begin{aligned}
\|f\|_{\widetilde{L}^{p(\cdot), \lambda(\cdot)}(X)} & \leq 2^{\frac{p_{+}+1}{p_{-}}}\left(\left\|f_{k}\right\|_{\widetilde{L}^{p(\cdot), \lambda(\cdot)}(X)}+\left\|f_{k}-f\right\|_{\widetilde{L}^{p(\cdot), \lambda(\cdot)(X)}}\right) \\
& \leq 2^{\frac{p_{+}+1}{p_{-}}}\left(M_{1}+\varepsilon\right)=: M .
\end{aligned}
$$

(b) We use

$$
|f(\cdot)-f(y)| \leq\left|f(\cdot)-f_{k}(\cdot)\right|+\left|f_{k}(\cdot)-f(y)\right|
$$

to have

$$
\begin{aligned}
& \left\|f_{B(\cdot, r)}|f(\cdot)-f(y)|^{q} \mathrm{~d} \mu(y)\right\|_{\widetilde{L}^{\frac{p(\cdot)}{q}, \lambda(\cdot)}(X)}^{1 / q} \\
& \quad \leq 2\left(\left\|\left(f_{B(\cdot, r)}\left|f(\cdot)-f_{k}(\cdot)\right|^{q} \mathrm{~d} \mu(y)+f_{B(\cdot, r)}\left|f(y)-f_{k}(\cdot)\right|^{q} \mathrm{~d} \mu(y)\right)\right\|_{\widetilde{L}^{\frac{p(\cdot)}{q}, \lambda(\cdot)}(X)}\right)^{\frac{1}{q}},
\end{aligned}
$$

where we have applied the inequality

$$
(a+b)^{q} \leq 2^{q}\left(a^{q}+b^{q}\right) \quad(a, b \geq 0) .
$$


We have

$$
\begin{aligned}
& \left\|f_{B(\cdot, r)}|f(\cdot)-f(y)|^{q} \mathrm{~d} \mu(y) \mid\right\|_{\widetilde{L}^{\frac{p(\cdot)}{q}, \lambda(\cdot)}(X)}^{1 / q} \\
& \leq 2^{1+\frac{p_{+}+q}{q p_{-}}}\left(\left\|f_{B(\cdot, r)}\left|f(\cdot)-f_{k}(\cdot)\right|^{q} \mathrm{~d} \mu(y)\right\|_{\widetilde{L}^{\frac{p(\cdot)}{q}, \lambda(\cdot)}(X)}\right)^{\frac{1}{q}} \\
& \left.\quad+\left\|f_{B(\cdot, r)}\left|f(y)-f_{k}(\cdot)\right|^{q} \mathrm{~d} \mu(y)\right\|_{\widetilde{L}^{\frac{p(\cdot)}{q}, \lambda(\cdot)}(X)}\right)\left|\left\|f_{f_{B(\cdot, r)}}\left|f(y)-f_{k}(\cdot)\right|^{q} \mathrm{~d} \mu(y)\right\|_{\widetilde{L}^{\frac{p(\cdot)}{q}, \lambda(\cdot)}(X)}^{\frac{1}{q}}\right)
\end{aligned}
$$

In the next step, we use

$$
\left|f_{k}(\cdot)-f(y)\right| \leq\left|f_{k}(y)-f_{k}(\cdot)\right|+\left|f_{k}(y)-f(y)\right|
$$

to obtain

$$
\left\|f_{B(\cdot, r)}|f(\cdot)-f(y)|^{q} \mathrm{~d} \mu(y)\right\|_{\widetilde{L}^{\frac{p(\cdot)}{q}, \lambda(\cdot)}(X)}^{1 / q} \leq 2^{2+\frac{1}{q}+\frac{2 p_{+}+2 q}{q p_{-}}}\left(\mathrm{I}_{1}+\mathrm{I}_{2}+\mathrm{I}_{3}\right) .
$$

Now, we give the bounds for $\mathrm{I}_{1}, \mathrm{I}_{2}$ and $\mathrm{I}_{3}$. Note that $\mathrm{I}_{1}<\varepsilon$ as we saw above.

Fix a point $x_{0} \in X$. Write $h_{k, R}:=\chi_{B\left(x_{0}, R\right)} \chi_{(0, R)}\left(\left|f_{k}\right|\right) f_{k}$. Since $f_{k} \in \widetilde{L}^{p(\cdot), \lambda(\cdot)}(X)$, by Lemma 3.3 , we have

$$
\lim _{R \rightarrow \infty}\left\|f_{k}-h_{k, R}\right\|_{L^{p(\cdot), \lambda(\cdot)(X)}}=0
$$

Thus, we can choose $R>0$, such that $\left\|f_{k}-h_{k, R}\right\|_{L^{p(\cdot), \lambda(\cdot)}(X)} \leq \epsilon$. We observe that

$$
\begin{aligned}
\mathrm{I}_{2}^{q} \leq & 4^{q+\frac{p_{+}+q}{p_{-}}}\left\|f_{B(\cdot, r)}\left|h_{k, R}(y)-f_{k}(y)\right|^{q} \mathrm{~d} \mu(y)\right\|_{\widetilde{L}^{\frac{p(\cdot)}{q}, \lambda(\cdot)}(X)} \\
& +4^{q+\frac{p_{+}+q}{p_{-}}}\left\|f_{B(\cdot, r)}\left|h_{k, R}(y)-h_{k, R}(\cdot)\right|^{q} \mathrm{~d} \mu(y)\right\|_{\widetilde{L}^{\frac{p(\cdot)}{q}, \lambda(\cdot)}(X)} \\
& +4^{q+\frac{p_{+}+q}{p_{-}}}\left\|f_{B(\cdot, r)}\left|f_{k}(\cdot)-h_{k, R}(\cdot)\right|^{q} \mathrm{~d} \mu(y)\right\|_{\widetilde{L}^{\frac{p(\cdot)}{q}, \lambda(\cdot)}(X)}
\end{aligned}
$$


Therefore

$$
\begin{aligned}
& \mathrm{I}_{2}^{q} \leq 4^{q+\frac{p_{+}+q}{p_{-}}}\left\|\left(\mathcal{M}_{q}\left(h_{k, R}-f\right)\right)^{q}\right\|_{\widetilde{L}^{\frac{p(\cdot)}{q}, \lambda(\cdot)}(X)} \\
& +4^{q+\frac{p_{+}+q}{p_{-}}}\left\|f_{B(\cdot, r)}\left|h_{k, R}(y)-h_{k, R}(\cdot)\right|^{q} \mathrm{~d} \mu(y)\right\|_{\widetilde{L}^{\frac{p(\cdot)}{q}, \lambda(\cdot)}(X)} \\
& +4^{q+\frac{p_{+}+q}{p_{-}}}\left\|\left|f-h_{k, R}\right|^{q}\right\|_{\widetilde{L}^{\frac{p(\cdot)}{q}, \lambda(\cdot)}(X)} \\
& \left.\leq 4^{q+\frac{p_{+}+q}{p_{-}}}\left(C^{q}+1\right)\left\|f_{k}-h_{k, R}\right\|_{\widetilde{L}^{p(\cdot), \lambda(\cdot)(X)}}^{q}\right) \\
& +4^{q+\frac{p_{+}+q}{p_{-}}}\left\|f_{B(\cdot, r)}\left|h_{k, R}(y)-h_{k, R}(\cdot)\right|^{q} \mathrm{~d} \mu(y)\right\|_{\widetilde{L}^{\frac{p(\cdot)}{q}, \lambda(\cdot)}(X)} .
\end{aligned}
$$

Since

$$
\lim _{r \rightarrow+0}\left\|f_{B(\cdot, r)}\left|h_{k, R}(y)-h_{k, R}(\cdot)\right|^{q} \mathrm{~d} \mu(y)\right\|_{\widetilde{L}^{\frac{p(\cdot)}{q}, \lambda(\cdot)}(X)}^{\frac{1}{q}}=0
$$

thanks to Lemma 3.7, for sufficiently small $r>0$, we have

$$
\left\|f_{B(\cdot, r)}\left|h_{k, R}(y)-h_{k, R}(\cdot)\right|^{q} \mathrm{~d} \mu(y)\right\|_{\widetilde{L}^{\frac{p(\cdot)}{q}, \lambda(\cdot)}(X)}^{\frac{1}{q}} \leq \epsilon .
$$

Therefore, we obtain $I_{2} \leq 4^{1+\frac{p_{+}+q}{p_{-}}}\left(C^{q}+2\right)^{\frac{1}{q}} \epsilon$ as long as $r$ is small enough. It remains to control $\mathrm{I}_{3}$. We have

$$
\begin{aligned}
& \mathrm{I}_{3}=\left\|f_{B(\cdot, r)}\left|f_{k}(y)-f(y)\right|^{q} \mathrm{~d} \mu(y)\right\|_{\widetilde{L}^{\frac{p(\cdot)}{q}, \lambda(\cdot)}(X)}^{\frac{1}{q}} \\
& =\left\|\left(f_{B(\cdot, r)}\left|f_{k}(y)-f(y)\right|^{q} \mathrm{~d} \mu(y)\right)^{\frac{1}{q}}\right\|_{\widetilde{L}^{p(\cdot), \lambda(\cdot)}(X)} \\
& \leq\left\|\mathcal{M}_{q}\left(f-f_{k}\right)\right\|_{\widetilde{L}^{p(\cdot), \lambda(\cdot)}(X)} \leq C\left\|f-f_{k}\right\|_{\widetilde{L}^{p(\cdot), \lambda(\cdot)}(X)} \leq C \varepsilon .
\end{aligned}
$$

(c) For $k=1, \ldots, N$, we fix sufficiently large $R_{k}$ satisfying

$$
\left\|f_{k} \chi_{X \backslash B\left(x_{0}, R_{k}\right)}\right\|_{\widetilde{L}^{p(\cdot), \lambda(\cdot)(X)}}<\varepsilon .
$$

Then, for $R=\max \left\{R_{k}: k=1, \ldots, N\right\}$, we have

$$
\begin{aligned}
& \left\|f \chi_{X \backslash B\left(x_{0}, R\right)}\right\|_{\widetilde{L}^{p(\cdot), \lambda(\cdot)}(X)} \\
& \quad \leq 2^{\frac{p_{+}+1}{p_{-}}}\left(\left\|\left(f-f_{k}\right) \chi_{X \backslash B\left(x_{0}, R\right)}\right\|_{\widetilde{L}^{p(\cdot), \lambda(\cdot)}(X)}+\left\|f_{k} \chi_{X \backslash B\left(x_{0}, R\right)}\right\|_{\widetilde{L}^{p(\cdot), \lambda(\cdot)}(X)}\right) \\
& \quad \leq 2^{\frac{p_{+}+1}{p_{-}}}\left(\left\|f-f_{k}\right\|_{\widetilde{L}^{p(\cdot), \lambda(\cdot)}(X)}+\left\|f_{k} \chi_{X \backslash B\left(x_{0}, R\right)}\right\|_{\widetilde{L}^{p(\cdot), \lambda(\cdot)}(X)}\right) \leq 2^{\frac{p_{+}+1}{p_{-}}+1} \varepsilon .
\end{aligned}
$$

We now show the reciprocal of the previous theorem. 
Theorem 3.9. Let $(X, \rho, \mu)$ be a bounded doubling metric measure space, and let $p(\cdot) \in \mathcal{P}(X, \mu)$ satisfy $0<p_{-} \leq p_{+}<\infty$ and $\sup _{x \in X} \frac{\lambda(x)}{p(x)}<\frac{1}{p_{+}}$. Assume that a subset $\mathcal{F}$ of $L^{p(\cdot), \lambda(\cdot)}(X)$ satisfies

(a) $\mathcal{F}$ is bounded in $L^{p(\cdot), \lambda(\cdot)}(X)$;

(b) for some $q \in\left(0, p_{-}\right)$

$$
\lim _{r \rightarrow+0} \sup _{f \in \mathcal{F}}\left\|f_{B(\cdot, r)}|f(\cdot)-f(y)|^{q} d \mu(y)\right\|_{L^{\frac{p(\cdot)}{q}, \lambda(\cdot)}(X)}=0 .
$$

Then, $\mathcal{F}$ is totally bounded in $L^{p(\cdot), \lambda(\cdot)}(X)$.

Proof. We divide the proof into two lemmata.

Lemma 3.10. The family $\mathcal{F}$ is $L^{p(\cdot), \lambda(\cdot)}(X)$-equi-integrable.

Proof. Let $q \in\left(0, p_{-}\right)$as in Theorem 3.9. Let $E$ be a measurable subset of $X$ and fix $r \in(0,1)$ and $x \in E$. Since $(a+b)^{q} \leq 2^{q}\left(a^{q}+b^{q}\right)$ for $a, b \geq 0$, we have

$$
|f(x)|^{q} \chi_{E}(x) \leq 2^{q}\left(|f(x)-f(y)|^{q} \chi_{E}(x)+|f(y)|^{q} \chi_{E}(x)\right)
$$

for any $y \in B(x, r)$ and $f \in \mathcal{F}$. Averaging the above inequality with respect to $y \in B(x, r)$, we obtain

$$
\begin{aligned}
& |f(x)|^{q} \chi_{E}(x) \\
& \quad \leq 2^{q}\left(f_{B(x, r)}|f(x)-f(y)|^{q} \mathrm{~d} \mu(y)+\chi_{E}(x) f_{B(x, r)}|f(y)|^{q} \mathrm{~d} \mu(y)\right) .
\end{aligned}
$$

By Hölder's inequality and Lemma 2.3, we have

$$
\begin{aligned}
\chi_{E} & (x) f_{B(x, r)}|f(y)|^{q} \mathrm{~d} \mu(y) \\
& \leq q\left(\frac{1}{p_{-}}+\frac{p_{+}-q}{p_{+}}\right) \frac{\chi_{E}(x)}{\mu(B(x, r))}\left\||f|^{q}\right\|_{L^{\frac{p(\cdot)}{q}}(B(x, r))}\|1\|_{L^{\frac{p(\cdot)}{p(\cdot)-q}}(B(x, r))} \\
& =q\left(\frac{1}{p_{-}}+\frac{p_{+}-q}{p_{+}}\right) \frac{\chi_{E}(x)}{\mu(B(x, r))}\|f\|_{L^{p(\cdot)}(B(x, r))}^{q}\|1\|_{L^{\frac{p(\cdot)}{p(\cdot)-q}(B(x, r))}} .
\end{aligned}
$$

By the definition of the Morrey norm $\|\cdot\|_{L^{p(\cdot) \lambda(\cdot)(X)}}$,

$$
\begin{aligned}
\|f\|_{L^{p(\cdot)}(B(x, r))} & \leq \sup _{\substack{x \in X \\
r \leq 1}} \mu(B(x, r))^{\frac{\lambda(x)}{p(x)}}\|f\|_{L^{p(\cdot), \lambda(\cdot)}(X)} \\
& \leq(\mu(X)+1)^{\sup _{x \in X} \frac{\lambda(x)}{p(x)}}\|f\|_{\widetilde{L}^{p(\cdot), \lambda(\cdot)}(X)} \\
& \leq(\mu(X)+1)^{\sup _{x \in X} \frac{\lambda(x)}{p(x)}} M,
\end{aligned}
$$

where in the last inequality, we applied the estimate $\|f\|_{\widetilde{L}^{p(\cdot), \lambda(\cdot)(X)}} \leq M$. Meanwhile, due to Lemma 2.1, we obtain

$$
\|1\|_{L^{\frac{p(\cdot)}{p(\cdot)-q}}(B(x, r))} \leq \max \left\{\mu(B(x, r))^{\frac{p_{+}-q}{p_{+}}}, \mu(B(x, r))^{\frac{p_{-}-q}{p_{-}}}\right\} .
$$


Therefore, thanks to $(2.5)$, there exists a constant $b>0$, such that

$$
\frac{1}{\mu(B(x, r))}\|1\|_{L^{\frac{p(\cdot)}{p(\cdot)-q}}(B(x, r))} \leq b^{-\frac{q}{p_{-}}} r^{-\frac{q s}{p_{-}}},
$$

provided that $r \ll 1$.

Thus, gathering the above estimates, we have

$$
\begin{aligned}
& \chi_{E}(x) f_{B(x, r)}|f(y)|^{q} \mathrm{~d} \mu(y) \\
& \quad \leq q\left(\frac{1}{p_{-}}+\frac{p_{+}-q}{p_{+}}\right) b^{-\frac{q}{p_{-}}} \chi_{E}(x)(\mu(X)+1)^{\sup _{z \in X} \frac{q \lambda(z)}{p(z)}} M^{q} r^{-\frac{q s}{p_{-}}} .
\end{aligned}
$$

Now, by (3.3), we have

$$
\begin{aligned}
& \left\|f \chi_{E}\right\|_{\widetilde{L}^{p(\cdot), \lambda(\cdot)}(X)}^{q} \\
& =\left\||f|^{q} \chi_{E}\right\|_{L^{\frac{p(\cdot)}{q}, \lambda(\cdot)}(X)} \\
& \leq C\left(\left\|f_{B(\cdot, r)}|f(\cdot)-f(y)|^{q} \mathrm{~d} \mu(y)\right\|_{L^{\frac{p(\cdot)}{q}, \lambda(\cdot)}(X)}+r^{-\frac{q s}{p_{-}}}\left\|\chi_{E}\right\|_{L^{\frac{p(x)}{q}, \lambda(\cdot)}(X)}\right),
\end{aligned}
$$

where $C:=2^{q+\frac{p_{+}+q}{p_{-}}}\left\{1+q\left(\frac{1}{p_{-}}+\frac{p_{+}-q}{p_{+}}\right) b^{-\frac{q}{p_{-}}}(\mu(X)+1)^{q \sup _{x \in X} \frac{\lambda(x)}{p(x)}} M^{q}\right\}$.

Finally, by the scaling relation, we obtain

$$
\begin{aligned}
& \left\|f \chi_{E}\right\|_{L^{p(\cdot), \lambda(\cdot)}(X)}^{q} \\
& \quad \leq C\left(\left\|f_{B(\cdot, r)}|f(\cdot)-f(y)|^{q} \mathrm{~d} \mu(y)\right\|_{L^{\frac{p(\cdot)}{q}, \lambda(\cdot)}(X)}+r^{-\frac{q s}{p-}}\left\|\chi_{E}\right\|_{L^{p(\cdot), \lambda(\cdot)}(X)}^{q}\right) .
\end{aligned}
$$

By condition (b) for given $\varepsilon>0$, we can and do fix sufficiently small $r>$ 0 , such that the first term on the right-hand side of (3.4) is less than $\varepsilon$. Thanks to Lemma 2.8 , we can choose $\delta \in(0,1)$, such that $r^{-\frac{q s}{p_{-}}}\left\|\chi_{E}\right\|_{L^{p(\cdot), \lambda(\cdot)}(X)}^{q} \leq$ $\varepsilon$ for any measurable set $E$ satisfying $\mu(E)<\delta$. Thus, the family $\mathcal{F}$ is $L^{p(\cdot), \lambda(\cdot)}(X)$-equi-integrable.

Lemma 3.11. The family $\mathcal{F}$ is totally bounded in $L^{0}(X)$.

Proof. Since the measure $\mu$ is doubling, $(X, \rho)$ is totally bounded (see [20]). Let us fix $\varepsilon \in(0,1)$. We take $\delta>0$, such that the following condition holds for all $f \in \mathcal{F}$ :

$$
\left\|f_{B(\cdot, 2 \delta)}|f(\cdot)-f(y)|^{q} \mathrm{~d} \mu(y)\right\|_{L^{\frac{p(\cdot)}{q}, \lambda(\cdot)}(X)}<\varepsilon^{\frac{q}{p_{-}}\left(p_{+}+1\right)} .
$$


Hence, in view of the Markov inequality and Lemma 2.1, we obtain

$$
\begin{aligned}
& \mu\left\{x \in X:\left(f_{B(x, 2 \delta)}|f(x)-f(y)|^{q} \mathrm{~d} \mu(y)\right)^{\frac{1}{q}}>\varepsilon\right\} \\
& \quad=\int_{\left\{x \in X:\left(f_{B(x, 2 \delta)}|f(x)-f(y)|^{q} \mathrm{~d} \mu(y)\right)^{\frac{1}{q}}>\varepsilon\right\}} \mathrm{d} \mu(x) \\
& \leq \int_{X}\left(\frac{1}{\varepsilon^{p(x)}}\left(f_{B(x, 2 \delta)}|f(x)-f(y)|^{q} \mathrm{~d} \mu(y)\right)^{\frac{p(x)}{q}} \mathrm{~d} \mu(x)\right) \\
& \leq \frac{1}{\varepsilon^{p}} \max \left\{\left\|f_{B(\cdot, 2 \delta)}|f(\cdot)-f(y)|^{q} \mathrm{~d} \mu(y)\right\|_{L^{\frac{p(\cdot)}{q}}(X)}^{\frac{p_{-}}{q}},\right. \\
& \left.\quad \times\left\|f_{B(\cdot, 2 \delta)}|f(\cdot)-f(y)|^{q} \mathrm{~d} \mu(y)\right\|_{L^{\frac{p(\cdot)}{q}}(X)}^{\frac{p_{+}}{q}}\right\}^{\cdot}
\end{aligned}
$$

By Lemma 2.7, one has

$\mu\left\{x \in X:\left(f_{B(x, 2 \delta)}|f(x)-f(y)|^{q} \mathrm{~d} \mu(y)\right)^{\frac{1}{q}}>\varepsilon\right\}<\max \left\{A^{\frac{p_{-}}{q}}, A^{\frac{p_{+}}{q}}\right\} \varepsilon$

where the constant $A$ is defined in Lemma 2.7. Next, for any $x, y \in X$ satisfying $\rho(x, y)<\delta$, we have

$$
\begin{aligned}
|f(x)-f(y)| \leq & 2^{1+\frac{1}{q}}\left(\left(f_{B(x, \delta)}|f(x)-f(z)|^{q} \mathrm{~d} \mu(z)\right)^{\frac{1}{q}}\right. \\
& \left.+\left(f_{B(x, \delta)}|f(y)-f(z)|^{q} \mathrm{~d} \mu(z)\right)^{\frac{1}{q}}\right) .
\end{aligned}
$$

A geometric observation shows that $B(x, \delta) \subset B(y ; 2 \delta)$. Since the measure $\mu$ is doubling, the last inequality implies that, for any $x, y \in X$ satisfying $\rho(x, y)<\delta$

$$
\begin{aligned}
|f(x)-f(y)| \leq & 2^{1+\frac{1}{q}} C_{d}^{-\frac{1}{q}}\left(\left(f_{B(x, 2 \delta)}|f(x)-f(z)|^{q} \mathrm{~d} \mu(z)\right)^{\frac{1}{q}}\right. \\
& \left.+\left(f_{B(y, 2 \delta)}|f(y)-f(z)|^{q} \mathrm{~d} \mu(z)\right)^{\frac{1}{q}}\right) .
\end{aligned}
$$


Let $\Lambda>1$. By virtue of the Markov inequality, Lemma 2.1, and the boundedness of $\mathcal{F}$ in $L^{p(\cdot), \lambda(\cdot)}(X)$, we have

$$
\begin{aligned}
\mu\{x & \in X:|f(x)|>\Lambda\} \\
& =\mu\left\{x \in X:|f(x)|^{p(x)}>\Lambda^{p(x)}\right\} \\
& \leq \frac{1}{\Lambda^{p_{-}}} \int_{X}|f(x)|^{p(x)} \mathrm{d} \mu(x) \\
& \leq \frac{1}{\Lambda^{p_{-}}} \max \left\{\|f\|_{L^{p(\cdot)}(X)}^{p_{-}} ;\|f\|_{L^{p(\cdot)}(X)}^{p_{+}}\right\} \\
& \leq \frac{\max \left\{A^{p_{-}}, A^{p_{-}}\right\}}{\Lambda^{p_{-}}} \max \left\{\|f\|_{L^{p(\cdot), \lambda(\cdot)(X)}}^{p_{-}} ;\|f\|_{L^{p(\cdot), \lambda(\cdot)}(X)}^{p_{+}}\right\} .
\end{aligned}
$$

Thanks to condition (a) of Theorem 3.8, there exists $M>0$ which is independent of $f \in \mathcal{F}$, such that

$$
\mu\{x \in X:|f(x)|>\Lambda\} \leq \frac{\max \left\{A^{p_{-}}, A^{p_{-}}\right\}}{\Lambda^{p_{-}}} \max \left\{M^{p_{-}} ; M^{p_{+}}\right\} .
$$

Therefore, if $\Lambda \gg 1$, then $\sup _{f \in \mathcal{F}} \mu\{x \in X:|f(x)|>\Lambda\}<\varepsilon$. We set

$$
\begin{aligned}
E(f):= & \{x \in X:|f(x)|>\Lambda\} \\
& \cup\left\{x \in X:\left(f_{B(x, 2 \delta)}|f(x)-f(y)|^{q} \mathrm{~d} \mu(y)\right)^{\frac{1}{q}}>\varepsilon\right\} .
\end{aligned}
$$

Then, $\mu(E(f))<\varepsilon$ as long as $\Lambda \gg 1$. Moreover

$$
\begin{gathered}
|f(x)| \leq \Lambda \text { for } x \in X \backslash E(f), \\
|f(x)-f(y)| \leq 2^{2+\frac{1}{q}} C_{d}^{-\frac{1}{q}} \varepsilon \text { for } x, y \in X \backslash E(f), \quad \rho(x, y)<\delta .
\end{gathered}
$$
$L^{0}(X)$.

Thus, by Theorem 2.10 , we conclude that $\mathcal{F}$ is totally bounded in

Now, we can finish the proof of the theorem. Indeed, we conclude from the previous lemmata that $\mathcal{F}$ is $L^{p(\cdot), \lambda(\cdot)}(X)$-equi-integrable, since $\mathcal{F}$ is totally bounded in $L^{0}(X)$. Thus, by virtue of Theorem 3.1, we obtain that $\mathcal{F}$ is totally bounded in $L^{p(\cdot), \lambda(\cdot)}(X)$.

\section{Examples and Remarks}

We summarize what we have obtained in the present paper together with some remarks. First, it was proved (see, e.g., [17,18]) that if $(X, \rho, \mu)$ is a doubling metric measure space with measure $\mu$ satisfying some additional regularity assumptions, $p(\cdot) \in \mathcal{P}_{\log }(X, \mu)$ satisfies $1<p_{-} \leq p_{+}<\infty$ and $0 \leq \lambda(x) \leq \min \left\{1, \frac{p(x)}{p_{\infty}}\right\}$ for all $x \in X$, then maximal operator $\mathcal{M}$ is bounded in $L^{p(\cdot), \lambda(\cdot)}(X)$. 
Example 4.1. Let $(X, \rho, \mu)$ be a metric measure space with finite measure, and let $p(\cdot)$ and $q(\cdot)$ be variable exponents satisfying $1 \leq p_{+}<q_{-} \leq q_{+}<\infty$. Let also $\lambda(\cdot)$ be a non-negative function. Assume that $s(x):=\frac{p(x) q(x)}{q(x)-p(x)}, x \in$ $X$ satisfies $\sup _{x \in X} \frac{\lambda(x)}{p(x)} \leq \frac{1}{s_{+}}$. Then, we claim that $L^{q(\cdot)}(X) \hookrightarrow L^{p(\cdot), \lambda(\cdot)}(X)$.

Indeed, by Lemmas 2.1 and 2.3, we have

$$
\begin{aligned}
\left\|f \chi_{B(x, t)}\right\|_{L^{p(\cdot)}(X)} & \leq C\|f\|_{L^{q(\cdot)}(X)}\left\|\chi_{B(x, t)}\right\|_{L^{\frac{p(\cdot) q(\cdot)}{q(\cdot)-p(\cdot)}(X)}} \\
& \leq C \max \left\{\mu(B(x, t))^{\frac{1}{s_{-}}}, \mu(B(x, t))^{\frac{1}{s_{+}}}\right\}\|f\|_{L^{q(\cdot)}(X)} .
\end{aligned}
$$

Thus

$$
\begin{aligned}
& \|f\|_{L^{p(\cdot), \lambda(\cdot)}(X)} \\
& =\sup _{\substack{x \in X \\
t>0}} \frac{1}{\mu(B(x, t))^{\frac{\lambda(x)}{p(x)}}}\left\|f \chi_{B(x, t)}\right\|_{L^{p(\cdot)}(X)} \\
& \leq C \sup _{\substack{x \in X \\
t>0}} \max \left\{\mu(B(x, t))^{\frac{1}{s_{-}}-\frac{\lambda(x)}{p(x)}}, \mu(B(x, t))^{\frac{1}{s_{+}}-\frac{\lambda(x)}{p(x)}}\right\}\|f\|_{L^{q(\cdot)}(X)} . \\
& \text { Since } \sup _{x \in X} \frac{\lambda(x)}{p(x)} \leq \frac{1}{s_{+}} \text {, it follows that: } \\
& \begin{array}{l}
\sup _{\substack{x \in X \\
t>0}} \max \left\{\mu(B(x, t))^{\frac{1}{s_{-}}-\frac{\lambda(x)}{p(x)}}, \mu(B(x, t))^{\frac{1}{s_{+}}-\frac{\lambda(x)}{p(x)}}\right\} \\
\quad \leq \sup _{x \in X} \max \left\{\mu(X)^{\frac{1}{s_{-}}-\frac{\lambda(x)}{p(x)}}, \mu(X)^{\frac{1}{s_{+}}-\frac{\lambda(x)}{p(x)}}\right\} \\
\quad \leq \max \left\{1, \mu(X)^{\left.\frac{1}{s_{-}}-\inf _{x \in X} \frac{\lambda(x)}{p(x)}\right\} .}\right.
\end{array}
\end{aligned}
$$

Thus, in total, we have $L^{q(\cdot)}(X) \hookrightarrow L^{p(\cdot), \lambda(\cdot)}(X)$.

From Theorems 3.8 and 3.9, we get the following.

Theorem 4.2. Let $(X, \rho, \mu)$ be a bounded doubling metric measure space. Let $q \in\left(0, p_{-}\right)$. Assume that $\sup _{x \in X} \frac{\lambda(x)}{p(x)}<\frac{1}{p_{+}}$, and that the powered maximal operator $\mathcal{M}_{q}: L^{p(\cdot), \lambda(\cdot)}(X) \rightarrow L^{p(\cdot), \lambda(\cdot)}(X)$.

Then, a family $\mathcal{F}$ of $\widetilde{L}^{p(\cdot), \lambda(\cdot)}(X)$ is totally bounded in $\widetilde{L}^{p(\cdot), \lambda(\cdot)}(X)$ if and only if the following conditions are satisfied:

(a) $\mathcal{F}$ is bounded in $\widetilde{L}^{p(\cdot), \lambda(\cdot)}(X)$; that is, there exists $M>0$, such that

$$
\sup _{f \in \mathcal{F}}\|f\|_{\widetilde{L}^{p(\cdot), \lambda(\cdot)}(X)} \leq M
$$

(b) $\lim _{r \rightarrow+0} \sup _{f \in \mathcal{F}}\left\|f_{B(\cdot, r)}|f(\cdot)-f(y)|^{q} d \mu(y)\right\|_{\widetilde{L}^{\frac{p(\cdot)}{q}, \lambda(\cdot)}(X)}=0$. 
Remark 4.3. Theorem 4.2 is new in the constant exponent case, but this question has also been considered in [5]. In [35], some sufficient conditions for subsets to be precompact sets in variable Morrey spaces. Note that in variable exponent Lebesgue spaces (that is for $\lambda(\cdot):=0$ ), Theorem 4.2 was proved in [3].

Moreover, thanks to Theorem 4.2, we can characterize precompact sets in $\widetilde{L}^{p(\cdot), \lambda(\cdot)}(X)$ in the case of $p_{-}>1$.

Theorem 4.4. Let $(X, \rho, \mu)$ be a bounded doubling metric measure space, and let $1<p_{-} \leq p_{+}<\infty$ and $\sup _{x \in X} \frac{\lambda(x)}{p(x)}<\frac{1}{p_{+}}$. Assume that the maximal operator $\mathcal{M}: L^{p(\cdot), \lambda(\cdot)}(X) \rightarrow L^{p(\cdot), \lambda(\cdot)}(X)$ is bounded.

Then, a family $\mathcal{F}$ of $\widetilde{L}^{p(\cdot), \lambda(\cdot)}(X)$ is totally bounded in $\widetilde{L}^{p(\cdot), \lambda(\cdot)}(X)$ if and only if the following conditions are satisfied:

(a) $\mathcal{F}$ is bounded in $\widetilde{L}^{p(\cdot), \lambda(\cdot)}(X)$;

(b) $\lim _{r \rightarrow+0} \sup _{f \in \mathcal{F}}\left\|f_{B(\cdot, r)}|f(\cdot)-f(y)| d \mu(y)\right\|_{\widetilde{L}^{p(\cdot), \lambda(\cdot)(X)}}=0$.

Let us close the paper with the following open question.

Open question: Let $(X, \rho, \mu)$ be an unbounded metric measure space. Characterize relatively compact sets in variable exponent Morrey spaces on $(X, \rho, \mu)$.

\section{Acknowledgements}

Some part of the paper has been performed during the visit of the second author to the Dumlupinar University. Moreover, the research of third author was partially supported by the grant of Cooperation Program 2532 TUBITAKRFBR (RUSSIAN foundation for basic research) (Agreement number no. 119N455). We wish to thank the referee for comments and suggestions. R.A. Bandaliyev and V.S. Guliyev were partially supported by the RUDN University Strategic Academic Leadership Program and by the grant of 1st Azerbaijan-Russia Joint Grant Competition (No. EIF-BGM-4-RFIF-1/201721/01M-M-08).

Open Access. This article is licensed under a Creative Commons Attribution 4.0 International License, which permits use, sharing, adaptation, distribution and reproduction in any medium or format, as long as you give appropriate credit to the original author(s) and the source, provide a link to the Creative Commons licence, and indicate if changes were made. The images or other third party material in this article are included in the article's Creative Commons licence, unless indicated otherwise in a credit line to the material. If material is not included in the article's Creative Commons licence and your intended use is not permitted by statutory regulation or exceeds the permitted use, you will need to obtain permission directly from the copyright holder. To view a copy of this licence, visit http:// creativecommons.org/licenses/by/4.0/.

Publisher's Note Springer Nature remains neutral with regard to jurisdictional claims in published maps and institutional affiliations. 


\section{References}

[1] D.R. Adams, Morrey Spaces, Lecture Notes in Applied and Numerical Harmonic Analysis, Birkhäuser, 2015

[2] Almeida, A., Hasanov, J.J., Samko, S.: Maximal and potential operators in variable exponent Lebesgue spaces. Georgian Math. J. 15(2), 195-208 (2008)

[3] Bandaliyev, R.A., Górka, P.: Relatively compact sets in variable-exponent Lebesgue spaces. Banach J. Math. Anal. 2(12), 331-346 (2018)

[4] Bandaliyev, R.A., Hasanov, S.G.: On denseness of $C_{0}^{\infty}(\Omega)$ and compactness in $L_{p(x)}$ for $0<p(x)<1$. Moscow Math. J. 1(18), 1-13 (2018)

[5] Bokayev, N.A., Burenkov, V.I., Matin, D.T.: On precompactness of a set in general local and global Morrey-type spaces. Eurasian Math. J. 8(3), 109-115 (2017)

[6] Caetano, A., Gogatishvili, A., Opic, B.: Compactness in quasi-Banach function spaces and applications to compact embeddings of Besov-type spaces. Proc. Edinb. Math. Soc. 146(5), 905-927 (2016)

[7] D. Cruz-Uribe, A. Fiorenza, Variable Lebesgue spaces. Foundations and Harmonic analysis, Birkhäuser/Springer, Heidelberg, 2013

[8] L. Diening, P. Harjulehto, P. Hästö, M. Rúžička, Lebesgue and Sobolev spaces with variable exponents, Springer Lecture Notes, v. 2017, Springer-Verlag, Berlin, 2011

[9] L. Diening, P. Hästö, A. Nekvinda, Open problems in variable exponent Lebesgue and Sobolev spaces, in: Proc. Int. Conference Function Spaces, Differential Operators and Nonlinear Analysis, Milovy, Czech Rep., May 28 - June 4, 2004, Math. Inst. Acad. Sci. Czech Rep., Prague, 2005, 38-58

[10] Górka, P., Macios, A.: Almost everything you need to know about relatively compact sets in variable Lebesgue spaces. J. Funct. Anal. 269, 1925-1949 (2015)

[11] Górka, P., Macios, A.: Riesz-Kolmogorov theorem on metric spaces. Miskolc Math. Notes 15(2), 459-465 (2014)

[12] P. Górka, P. Pośpiech, Banach function spaces on locally compact groups.Ann. Funct. Anal., https://doi.org/10.1215/20088752-2019-0002.

[13] P. Górka, H. Rafeiro, From Arzelà-Ascoli to Riesz-Kolmogorov, Nonlinear Anal. 144 (2016), 23-31; Correction, Nonlinear Anal. 149 (2017), 177-179

[14] Górka, P., Rafeiro, H.: Light side of compactness in Lebesgue spaces: Sudakov theorem. Ann. Acad. Sci. Fenn. Math. 42(1), 135-139 (2017)

[15] Guliyev, V.S., Hasanov, J.J., Samko, S.G.: Boundedness of the maximal, potential and singular operators in the generalized variable exponent Morrey spaces. Math. Scand. 107, 285-304 (2010)

[16] Guliyev, V.S., Hasanov, J.J., Samko, S.G.: Maximal, potential and singular operators in the local complementary variable exponent Morrey type spaces. J. Math. Anal. Appl. 401(1), 72-84 (2013)

[17] Guliyev, V.S., Samko, S.G.: Maximal, potential and singular operators in the generalized variable exponent Morrey spaces on unbounded sets. J. Math. Sci. 193(2), 228-248 (2013)

[18] Guliyev, V.S., Samko, S.G.: Maximal operator in variable exponent generalized Morrey spaces on quasi-metric measure space. Mediterr. J. Math. 13, 1151-1165 (2016) 
[19] Harjulehto, P., Hästö, P.: Variable exponent Lebesgue spaces on metric spaces: Hardy-Littlewood maximal operator. Real Anal. Exchange 30, 87-104 (2004)

[20] J. Heinonen, Lectures on analysis on metric spaces, Universitext, Springer, New York, 2001

[21] M. Izuki, E. Nakai, Y. Sawano, Function spaces with variable exponents-an introduction, Sci. Math. Jpn. 77(2014), no. 2, 187-315

[22] A. N. Kolmogorov, Über Kompaktheit der Funktionenmengen bei der Konvergenz im Mittel, in Selected works of A. N. Kolmogorov, Vol. I, Kluwer, Dordrecht, 1991, 147-150

[23] V.G. Krotov, Compactness criteria in the spaces Lp, p> 0 (in Russian), Mat. Sb. 203 (2012), no. 7, 129-148; English translation in Sb. Math. 203 (2012), no. $7-8,1045-1064$

[24] Li, F., Li, Z., Pi, L.: Ling, Variable exponent functionals in image restoration. Appl. Math. Comput. 216(3), 870-882 (2010)

[25] Mizuta, Y., Shimomura, T.: Sobolev embeddings for Riesz potentials of functions in Morrey spaces of variable exponent. J. Math. Soc. Jpn. 60, 583-602 (2008)

[26] Morrey, C.B.: On the solutions of quasi-linear elliptic partial differential equations. Trans. Am. Math. Soc. 43, 126-166 (1938)

[27] Orlicz, W.: Über konjugierte exponentenfolgen. Studia Math. 3, 200-212 (1931)

[28] Peetre, J.: On the theory of $\mathcal{L}_{p, \lambda}$ spaces. J. Funct. Anal. 4, 71-87 (1969)

[29] Rafeiro, H.: Kolmogorov compactness criterion in variable exponent Lebesgue spaces. Proc. A. Razmadze Math. Inst. 150, 105-113 (2009)

[30] Riesz, M.: Sur les ensembles compacts de fonctions sommables. Acta. Szeged Sect. Math. 6, 136-142 (1933)

[31] M. Růẑiĉka, Electrorheological Fluids: Modeling and Mathematical Theory, Springer-Verlag, Berlin, 2000

[32] Samko, S.: On a progress in the theory of Lebesgue spaces with variable exponent: maximal and singular operators. Integr. Transf. Spec. Funct. 16, 461-482 (2005)

[33] Y. Sawano, G. Di Fazio, D. I. Hakim, Morrey Spaces: Introduction and Applications to Integral Operators and PDE's, CRC Press, 2020

[34] Sawano, Y., Tanaka, H.: The Fatou property of block spaces. J. Math. Sci. Univ. Tokyo 22, 663-683 (2015)

[35] W. Wang, J. Xu, Precompact sets, boundedness, and compactness of commutators for singular integrals in variable Morrey spaces, J. Funct. Spaces, 2017 (2017), Article ID 3764142, 1-9

[36] V.V. Zhikov, Averaging of functionals of the calculus of variations and elasticity theory, Math. USSR-Izv. (29) (1987), 675-710 
Rovshan A. Bandaliyev and Vagif S. Guliyev

People Friendship University of Russia (RUDN University)

6 Maklaya st

Moscow 117198

Russian Federation

e-mail: bandaliyevr@gmail.com

Vagif S. Guliyev

e-mail: vagif@guliyev.com

and

Institute of Mathematics and Mechanics of NAS of Azerbaijan

Baku

Azerbaijan

Przemysław Górka

Department of Mathematics and Information Sciences

Warsaw University of Technology

Ul. Koszykowa 75

Warsaw 00-662

Poland

e-mail: pgorka@mini.pw.edu.pl

Vagif S. Guliyev

Department of Mathematics

Dumlupinar University

Kutahya

Turkey

and

Institute of Applied Mathematics

Baku State University

Baku

Azerbaijan

Yoshihiro Sawano

Department of Mathematics and Information Science

Tokyo Metropolitan University

Hachioji-shi Tokyo

Japan

e-mail: yoshihiro-sawano@celery.ocn.ne.jp

Received: August 13, 2020.

Revised: November 27, 2020.

Accepted: August 1, 2021. 